\title{
A theory-based implementation program for alcohol screening and brief intervention (ASBI) in general practices: Planned development and study protocol of a cluster randomised controlled trial
}

Citation for published version (APA):

Abidi, L., Oenema, A., Candel, M., \& van de Mheen, D. (2016). A theory-based implementation program for alcohol screening and brief intervention (ASBI) in general practices: Planned development and study protocol of a cluster randomised controlled trial. Contemporary Clinical Trials, 51, 78-87. https://doi.org/10.1016/j.cct.2016.10.008

Document status and date:

Published: 01/11/2016

DOI:

10.1016/j.cct.2016.10.008

Document Version:

Publisher's PDF, also known as Version of record

Document license:

Taverne

Please check the document version of this publication:

- A submitted manuscript is the version of the article upon submission and before peer-review. There can be important differences between the submitted version and the official published version of record. People interested in the research are advised to contact the author for the final version of the publication, or visit the DOI to the publisher's website.

- The final author version and the galley proof are versions of the publication after peer review.

- The final published version features the final layout of the paper including the volume, issue and page numbers.

Link to publication

\footnotetext{
General rights rights.

- You may freely distribute the URL identifying the publication in the public portal. please follow below link for the End User Agreement:

www.umlib.nl/taverne-license

Take down policy

If you believe that this document breaches copyright please contact us at:

repository@maastrichtuniversity.nl

providing details and we will investigate your claim.
}

Copyright and moral rights for the publications made accessible in the public portal are retained by the authors and/or other copyright owners and it is a condition of accessing publications that users recognise and abide by the legal requirements associated with these

- Users may download and print one copy of any publication from the public portal for the purpose of private study or research.

- You may not further distribute the material or use it for any profit-making activity or commercial gain

If the publication is distributed under the terms of Article 25fa of the Dutch Copyright Act, indicated by the "Taverne" license above,

Download date: 26 Apr. 2023 


\title{
A theory-based implementation program for alcohol screening and brief intervention (ASBI) in general practices: Planned development and study protocol of a cluster randomised controlled trial
}

\author{
L. Abidi ${ }^{\text {a,* }}$, A. Oenema ${ }^{\text {a }}$, M.J.J.M. Candel ${ }^{\text {b }}$, D. van de Mheen ${ }^{\text {a,c,d }}$ \\ a Department of Health Promotion, School of Public Health and Primary Care, Maastricht University, PO Box 616, 6200 MD Maastricht, The Netherlands \\ b Department of Methodology \& Statistics, School of Public Health and Primary Care, Maastricht University, PO Box 616, 6200 MD Maastricht, The Netherlands \\ c IVO Addiction Research Institute, Rotterdam, The Netherlands \\ d Erasmus Medical Centre, Rotterdam, The Netherlands
}

\section{A R T I C L E I N F O}

\section{Article history:}

Received 4 July 2016

Received in revised form 19 October 2016

Accepted 22 October 2016

Available online 24 October 2016

\section{Keywords:}

Implementation

Alcohol consumption

Screening

Brief intervention

\begin{abstract}
A B S T R A C T
Background: Previous studies have shown that alcohol screening and brief intervention (ASBI) in general practices can lead to significant reductions in alcohol consumption among patients, yet ASBI is rarely implemented into routine clinical practice. The aim of this paper is to describe the development and evaluation of an ASBI implementation program aimed at increasing ASBI delivery rates of general practitioners (GPs) and decreasing patients' alcohol consumption.

Methods/design: This study protocol describes the step-wise development and evaluation of an ASBI implementation program. A four-step method is used to identify relevant determinants of change and intervention components based on the Behaviour Change Wheel and the Theoretical Domains Framework. The program will be evaluated in general practices in The Netherlands in a two-arm cluster randomised controlled trial which investigates the effect of the program on GPs' ASBI delivery behaviour as well as on patients' alcohol consumption. Discussion: Effective theory- and practice-based strategies to implement ASBI in general practices are highly needed. Using a stepwise method we described the development of a program consisting of an e-learning module, a tailored feedback module and environmental support and materials. We hypothesize that this program will result in an increase of GPs' ASBI delivery behaviour. Secondly, we expect an overall decrease in percentage of patients with excessive or problematic alcohol use and a higher proportion of patients from GPs receiving the ASBI implementation program decreasing their alcohol consumption, compared to patients from GPs in the control group.

Trial registration: NTR5539
\end{abstract}

(c) 2016 Elsevier Inc. All rights reserved.

\section{Introduction}

Alcohol use has been related to more than 200 diseases, injuries and other health conditions [1] and is one of the most important risk factors for ill-health and premature death [2]. In the Netherlands, more than $10 \%$ of the population aged 16 to 69 years reported drinking alcohol at levels considered excessive or problematic [3]. Excessive alcohol use is defined as drinking more than the recommended maximum of two units of alcohol per day for men and one unit of alcohol per day for women and having at least two drinking-free days per week. Problematic alcohol use is defined as a drinking pattern leading to physical and/ or psychological or social problems and preventing adequate treatment of ongoing problems [4]. Currently, about 70 to $90 \%$ of excessive or

\footnotetext{
* Corresponding author.

E-mail address: Latifa.abidi@maastrichtuniversity.nl (L. Abidi).
}

problematic alcohol users are not recognized as such by their general practitioner (GP) [5].

The Dutch Guidelines for General Practitioners recommend GPs to screen patients on their alcohol use when they present with early signs, such as unexplained somatic symptoms, gastro-intestinal problems or psychological symptoms such as depression or anxiety [4]. The scientific literature has provided robust evidence showing that alcohol screening and brief intervention (ASBI) in primary care lead to significant reductions of alcohol consumption [6,7] as well as improved health outcomes for patients, together with savings to health care resources $[7,8]$. However, the impeded delivery of ASBI in clinical practice reflects an incomplete understanding of how to translate those effects to real-world clinical practice and how to bring about wide-spread implementation of ASBI [9]. Moreover, there is a lack of evidence-based implementation interventions and a lack of insight into which methods are suitable to facilitate implementation. 
The difficulty in involving GPs in addressing excessive and problematic alcohol use of their patients has been attributed to a variety of barriers, such as lack of knowledge about early signs and risk groups, fear of antagonizing patients and a lack of time $[5,10,11]$. Although, a recent meta-analysis recommends implementation interventions to encompass multifaceted strategies [12], a previous trial evaluating an implementation program consisting of patient-, professional- and organisation-orientated strategies, proved too extensive and ineffective [13]. The trial also failed to show an effect on patient alcohol consumption, highlighting the difficulty of translating efficacy trial effects into real-world clinical practice. These previous null-findings underscore the need for theory-based as well as practice-based approaches to ASBI implementation [14]. A key challenge, therefore, is to develop an implementation intervention fitting to the context of GP practices that targets the identified barriers using theory-based strategies.

Building upon the identified gaps, an implementation program has been developed aimed at increasing ASBI delivery by GPs as well as decreasing patients' alcohol use. The aim of this paper is [1] to describe the step-wise development of the ASBI implementation program and [2] to describe the study design of the cluster randomised controlled trial testing the effectiveness of the program in terms of ASBI delivery behaviour of GPs and subsequent alcohol consumption rates among their patients.

\section{Methods/design}

The development of implementation interventions requires a systematic step-wise approach with a strong rationale, in which the target points are identified, effective methods are linked and intervention components are made explicit [14]. We used a four step approach as described by French et al. 2012 [14]. First we have identified and specified the target behaviour (i.e. delivery of ASBI). Second, relevant determinants of change were identified. Third, intervention components were identified by linking determinants to effective behaviour change techniques and feasible modes of delivery were selected. Fourth, behaviour change is measured and evaluated in a randomised controlled trial.

\subsection{Step 1: specifying the target behaviour}

The specified target behaviour for ASBI is based upon the NHG Guideline Problematic Alcohol Use from the Dutch College of General Practitioners [4]. The core elements can be presented as follows:

1. GPs inquire about alcohol when patients present with the following three symptoms as potential early signs (symptom-specific screening): [1] frequent visits and a changing, unclear pattern of symptoms or unexplained somatic symptoms, [2] gastro-intestinal problems, [3] psychological symptoms (e.g. depression or anxiety), combined with sleeping problems.

2. GP's provide brief advice (of max. 5 to 10 minutes) or referral to the practice nurse mental health, primary care psychologist, or specialized addiction care (secondary care) in case of excessive or problematic alcohol use.

Given that GPs find symptom-specific rather than universal screening the most acceptable method of identifying alcohol-related risks [18], we chose to focus on symptom-specific screening.

\subsection{Step 2: identifying barriers and facilitators}

The second step is to understand which factors, or 'determinants,' would impede or facilitate ASBI delivery. In order to identify all barriers associated with ASBI, the ASBI implementation literature was explored $[5,10,11]$. The identified different factors associated with ASBI were examined by three of the authors (LA, AO and DM) who agreed on a list of relevant factors (Table 1 ).

Subsequently these barriers had to be categorized into a theoretical framework. The Behaviour Change Wheel (BCW) was used for this. The
BCW is an applicable model to analyse underlying factors of a target behaviour. At the centre of the BCW is a system consisting of three components: Capability, Opportunity, and Motivation which interact to generate Behaviour (COM-B). The COM-B system provides a comprehensive explanation of behaviour by including automatic processes, and explicitly including factors at the person's level (capability and motivation) as well as on the organisational level (opportunity). We mapped the identified factors into the COM-B components. Table 1 shows how factors extracted from the literature map into the COM-B system.

The next action was to refine the identified barriers into more specific theoretical constructs, in order to link evidence-based behaviour change techniques to theoretical constructs in the next step. To pinpoint the theoretical constructs of the identified barriers, we used the TDF. The TDF is an integrative framework developed from a synthesis of psychological theories as a tool to map theoretically derived behavioural determinants to behaviour change techniques. The TDF consists of 12 domains, such as knowledge, cognitive and interpersonal skills, beliefs

Table 1

Barriers to ASBI implementation ordered by the COM-B system.

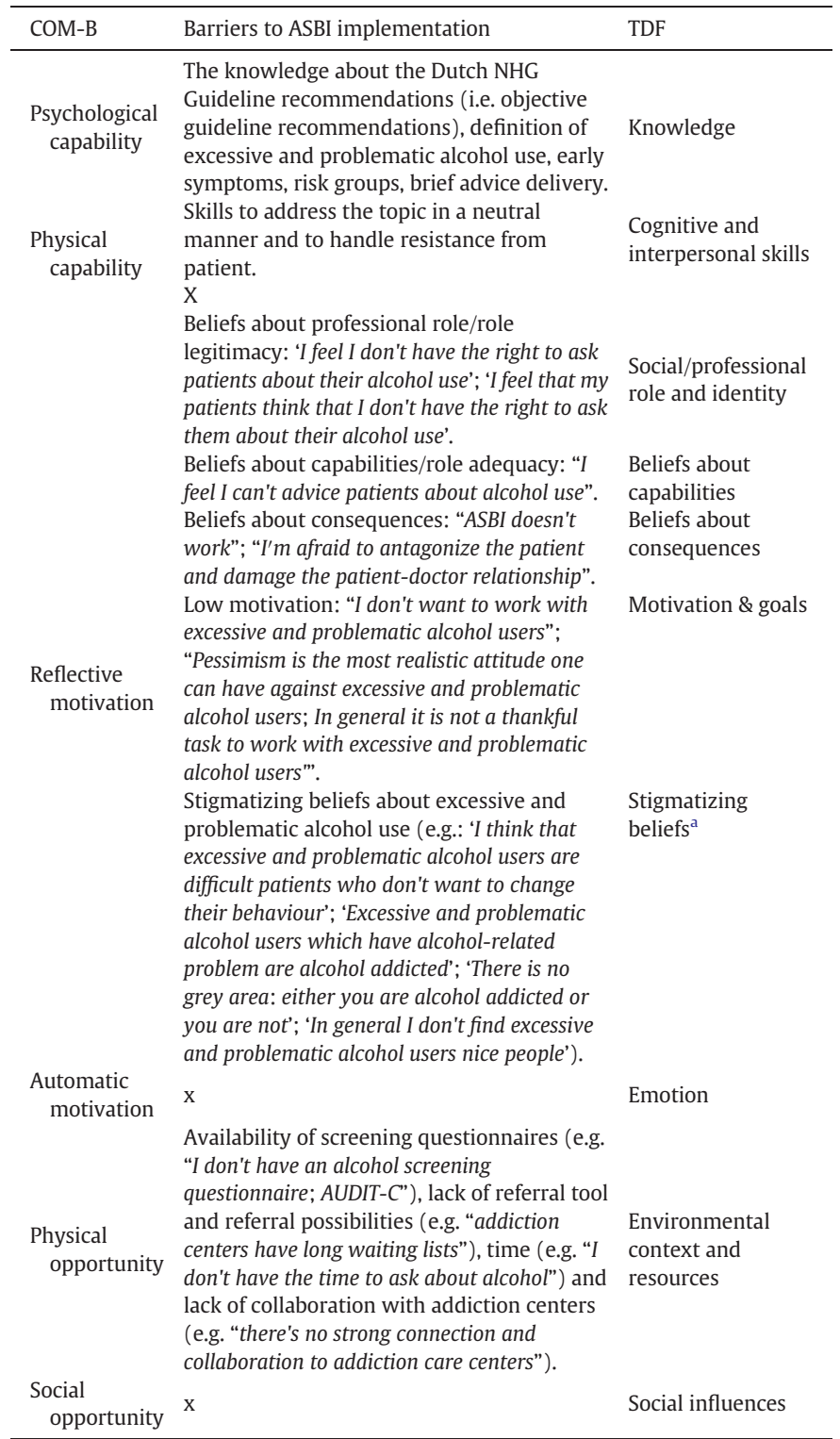

Note: COM-B = Capability, Opportunity, Motivation - Behaviour; TDF = Theoretical Domains Framework [17].

a Stigmatizing beliefs was added as an extra theoretical domain. 
Table 2

Content of Implementation program and behaviour change techniques by relevant domains of the Theoretical Domains Framework.

Barriers
1. Not knowing the definition of excessive and
problematic alcohol use.
2. Lack of knowledge about early signs.
3. Lack of knowledge about patient risk groups.
4. Lack of knowledge about screening
questionnaires.
5. Lack of knowledge about brief advice delivery
(i.e. objective guideline recommendations).

1. Lack of skills to address the topic when suspecting problematic alcohol use.

2. Lack of skills to handle resistance from patient.

$\begin{array}{ll}\text { TDF domain } & \begin{array}{l}\text { Behaviour change } \\ \text { techniques }\end{array} \\ & \\ \text { Knowledge } & \begin{array}{l}\text { 1) Information regarding } \\ \text { behaviour and outcome }\end{array}\end{array}$

Cognitive and interpersonal skills
1) Specifying goal/target
2) Monitoring
3) Self-monitoring
4) Rewards/incentives
5) Graded-task: starting
with easy tasks
6) Increasing skills: goal setting
7) Rehearsal of relevant
skills
8) Modelling/demonstration
of behaviour by others.
9) Homework
10) Perform behaviour in
different settings

Beliefs about professional role/role legitimacy:

1. 'I feel I don't have the right to ask patients about their alcohol use'.

2. 'I feel that my patients think that I don't have the right to ask them about their alcohol use'.

Beliefs about capabilities/role adequacy:

1. "I feel I can't advice patients about alcohol".

\author{
Beliefs about consequences: \\ 1. "ASBI doesn't work" \\ 2. "I'm afraid to antagonize the patient and \\ damage the patient-doctor relationship".
}

\footnotetext{
Low motivation to work with problematic alcohol users:

1. "I don't want to work with excessive and problematic alcohol users".

2. 'Pessimism is the most realistic attitude one can have against excessive and problematic alcohol users'.

3. 'In general it is not a thankful task to work with excessive and problematic alcohol users'.
}

\footnotetext{
1. Availability of screening questionnaires

2. Referral possibilities

3. Time

4. Collaboration with addiction centers.
}

Social/professional role and identity

1) Social processes of encouragement, pressure and support.

Beliefs about capabilities

Beliefs about consequences

Motivations and goals

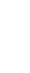

\section{1) Self-monitoring \\ 2) Graded-task: starting \\ with easy tasks}

setting

4) Coping skills

5) Rehearsal of relevant

skills

6) Social processes of

encouragement, pressure,

support

7) Feedback

8) Self-talk

1) Self-monitoring

2) Persuasive

communication

behaviour and outcome

4) Feedback

1) Specifying goal/target

2) Contract

3) Rewards/incentives

4) Graded-task: starting

with easy tasks

5) Increasing skills: goal

setting

encouragement, pressure,

support

7) Persuasive

communication

8) Information regarding

behaviour, outcome

Environmental

context and

resources behaviour)
3) Information regarding

6) Social processes of
3) Increasing skills: goal

Intervention components

Mode: E-learning module

Technique: Education

Content: The E-learning module focusses on the following goals:

1. Knowing the definition of excessive and problematic alcohol use.

2. Knowing the early signs \& symptoms related to problematic alcohol use.

3. Knowing the epidemiology and risk groups of problematic alcohol use.

4. Knowing the AUDIT-C test.

5. Knowing how to deliver brief advice according to the NHG Guideline recommendations.

Mode: E-learning module

Technique: Modelling/demonstration of behaviour by others Content: The E-learning module focusses on the following goals:

1. Knowing how to address the topic and discuss alcohol use.

2. Knowing how to handle resistance from patient.

Mode: Tailored feedback module

Technique: Encouragement

Content: The feedback module focusses on the following goals:

1. Increasing role legitimacy through encouragement by providing

feedback about GPs and practice nurses views on the important roles of GPs in early detection and treatment of problematic alcohol use

2. Increasing role legitimacy through encouragement by providing feedback about patient's positive views on discussing alcohol use with their GP.

Mode: Tailored feedback module

Technique: Encouragement

Content: The feedback module focusses on the following goals:

1. Increasing task-specific self-esteem through feedback by providing tips and encouragement (e.g. depiction of an easy step-wise method of how to advice patients on alcohol use).

Mode: Tailored feedback module

Technique: Feedback, Persuasive communication; Information regarding behaviour and outcome.

Content: The feedback module focusses on the following goals:

1. Increasing positive beliefs about ASBI effectiveness.

2. Decreasing fear to antagonize the patient.

Mode: Tailored feedback module

Technique: Persuasive communication, Information regarding

behaviour, outcome.

Content: The feedback module targets motivation to work with problematic alcohol users and focusses on the following goals:

1. Increasing motivation to work with problematic alcohol users by targeting the negative belief about these patients (e.g. "In general, patients are reactive to advice from GPs").

2. Emphasizing that it can be rewarding to work with excessive and problematic alcohol users.

Mode: Materials, tailored feedback module \& strengthening of connections with addiction care centers.

1) Environmental changes Technique: Environmental changes and support; Information provision.

(e.g. objects to facilitate Content: The following materials will be provided:
1. The AUDIT-C screening questionnaire with instructions.

2. A referral scheme.

3. Information provision about the time-efficiency of ASBI. 
Table 2 (continued)

\begin{tabular}{|c|c|c|c|}
\hline Barriers & TDF domain & $\begin{array}{l}\text { Behaviour change } \\
\text { techniques }\end{array}$ & Intervention components \\
\hline & & & $\begin{array}{l}\text { 4. An overview of the contact information of contact-persons in local } \\
\text { addiction care centers. }\end{array}$ \\
\hline $\begin{array}{l}\text { 1. 'I think that excessive and problematic alcohol } \\
\text { users are difficult patients who don't want to } \\
\text { change their behaviour' } \\
\text { 2. 'Excessive and problematic alcohol users which } \\
\text { have alcohol-related problem are alcohol addicted' } \\
\text { 3. 'There is no grey area: either you are alcohol } \\
\text { addicted or you are not'; } \\
\text { 4. 'In general I don't find excessive and problematic } \\
\text { alcohol users nice people'. }\end{array}$ & $\begin{array}{l}\text { Stigmatizing } \\
\text { beliefs }^{\mathrm{a}}\end{array}$ & $\mathrm{x}$ & $\begin{array}{l}\text { Mode: Tailored feedback module } \\
\text { Technique: Persuasive communication } \\
\text { Content: The feedback module targets the stigma on excessive and } \\
\text { problematic alcohol use and focusses on the following goal: } \\
\text { 1. De-stigmatization of excessive and problematic alcohol use. }\end{array}$ \\
\hline
\end{tabular}

Note: BCT column enlists all possible techniques; chosen BCTs are enlisted under "Intervention components".

a Stigmatizing beliefs was added as an extra theoretical domain.

about consequences, motivation and goals, with each domain having a set of theoretical constructs that had been identified as components. Applying the TDF framework to our analysis of ASBI behaviour, a total of 8 out of the 12 domains concern characteristics of the GPs or practices which play a role in ASBI implementation (see Table 2). Stigmatizing beliefs was added as an extra theoretical domain. Therefore, we will focus on modifying 8 theoretical domains in the next step of developing the implementation intervention.

\subsection{Step 3a: linking behaviour change techniques to barriers and facilitators}

To inform the selection of behaviour change techniques that target the barriers described in the previous step, we used the TDF as a practical tool for selecting appropriate behaviour change techniques [19]. The approach of mapping behaviour change techniques to TDF domains has been done in previous studies and incorporated into protocols for the development of complex interventions $[14,20]$. This step resulted in the selection of a number of behaviour change strategies to modify the barriers identified in the previous step (see Table 2):

- Knowledge: Information provision regarding ASBI behaviour is the behaviour change technique used to modify knowledge barriers. More specifically, information is provided about: [1] the definition of problematic alcohol use and excessive alcohol use, [2] symptomatology and early symptoms of problematic alcohol use, [3] risk-groups, [4] measurement instruments (i.e. knowing how to use the AUDIT-C), [5] interventions/techniques (e.g. knowing about stadia of behaviour change; knowing how to listen and to ask open questions, knowing brief interventions such as brief advice).

- Cognitive and interpersonal skills: Cognitive and interpersonal barriers (i.e.: how to address the topic of alcohol use, diagnosing (knowing which questions to ask to assess the problem), and how to handle resistance from patients) are targeted by modelling/demonstration of behaviour by others.

- Beliefs about professional role: Barriers concerning role legitimacy (i.e.: 'I feel I don't have the right to ask patients about their alcohol use'; 'I feel that my patients think that I don't have the right to ask them about their alcohol use') are targeted through encouragement by providing evidence about patient's positive views on discussing alcohol use with their GP. Moreover, feedback will be provided about GPs and practice nurses views on the important roles of GPs in early detection and treatment of problematic alcohol use.

- Beliefs about capabilities: The barrier about role adequacy (i.e.: "I feel I can't advice patients about alcohol") will be targeted by providing GPs with tips and encouragement (e.g. depiction of an easy step-wise method of how to advice patients on alcohol use).

- Motivation and goals: Persuasive communication and information provision are used to target barriers about motivation (i.e.: 'I don't want to work with excessive and problematic alcohol users'; 'Pessimism is the most realistic attitude one can have against excessive and problematic alcohol users'; 'In general it is not a thankful task to work with excessive and problematic alcohol users').

- Beliefs about consequences: Barriers related to beliefs about consequences (i.e. "ASBI doesn't work"; "I'm afraid to antagonize the patient and damage the patient-doctor relationship") are targeted by increasing positive beliefs about ASBI effectiveness and decreasing fear to antagonize the patient through persuasive communication and feedback.

- Stigmatizing beliefs: Persuasive communication targets the following stigma beliefs on problematic alcohol use: 'I think that excessive and problematic alcohol users are difficult patients who don't want to change their behaviour'; 'Excessive and problematic alcohol users which have alcohol-related problem are alcohol addicted'; 'There is no grey area: either you are alcohol addicted or you are not'; 'In general I don't find excessive and problematic alcohol users nice people'.

- Environmental context and resources: Barriers related to environmental context and resources (i.e.: availability of screening questionnaires, lack of referral tool and referral possibilities, and lack of collaboration with addiction centers) are targeted by providing materials (i.e.: screening questionnaires, a stepwise ASBI scheme, a referral tool and an informative website) and supportive contacts from addiction prevention centers for GPs. Addiction prevention workers in addiction care centers will be connected to general practices in the local region, aiming to provide environmental support in conducting ASBI.

- Behaviour: In addition to the above mentioned barriers, GPs receive tailored feedback about their ASBI behaviour. More specifically, an assessment is made about whether they conduct symptom-specific screening and whether they provide patients with brief advice or referral if needed, after which tailored advice will be provided about how their ASBI behaviour can be improved.

2.4. Step 3b: what are feasible, locally relevant, and acceptable modes of delivery?

To translate ASBI to an acceptable yet adequate format, the following modes of delivery (Table 2) were chosen based on results of a Delphistudy in which applicable solutions to the ASBI-barriers were identified by health professionals and addiction prevention experts [18]:

\subsubsection{E-learning module}

E-learning was identified as an alternative approach to face-to-face learning and consensually rated as an applicable strategy to enhance knowledge by GPs [18]. Therefore, we chose e-learning as a mode of delivery rather than face-to-face trainings. Internet-based learning has 
been shown to be at least as effective as traditional learning methods [21], while enabling GPs to participate at a time and place convenient to them $[22,23]$. The e-learning module contains information provision and modelling/demonstration of behaviour by peers as strategies to target behaviour.

\subsubsection{Online tailored feedback module}

An online feedback module was chosen as the mode of delivery to address barriers related to knowledge and motivation. An online format provides us with the opportunity to tailor feedback to the individual participant using computer algorithms. Individual computerized tailoring is a technique that produces personally relevant messages for each participant [24]. The use of tailored information, rather than generic information has been proven to be an effective strategy in numerous studies $[25,26]$. Moreover, similar as e-learning, an online feedback module provides GPs with the flexibility to participate at a time and place convenient to them. The feedback module contains information provision, persuasive communication and tailored feedback as strategies to target, among others, motivation, beliefs about professional role, beliefs about capabilities, beliefs about consequences, and stigmatizing beliefs.

\subsubsection{Website to access support and materials}

To provide GPs with contact information of addiction prevention workers, we chose to develop an online overview of local addiction prevention workers on the study website. The use of an informative website has been consensually rated as an applicable enabling strategy for ASBI implementation by GPs [18]. An advantage is that information can easily be updated and made available to GPs. Addiction prevention workers will be connected to general practices in the local region, aiming to provide environmental support in conducting ASBI to GPs. Furthermore these contacts aim to provide consultation to GPs about prevention care and interventions for patients. The format of the supportive materials is partly online (i.e. a step-wise ASBI scheme and a referral scheme) and partly on paper (AUDIT-C screening questionnaires).

\subsection{Step 3c: development of the program}

We chose to use an existing e-learning module, developed (among others) by the Ministry of Health, Welfare and Sport, Lundbeck and Partnership Early detection Alcohol [27]. The first session of the e-learning module, one hour duration, consists of role-play videos with different types of patients and scenarios in which GPs ask their patients about alcohol and then provide a brief intervention. The second session of one hour duration consists of a dialogue session between health professionals in which epidemiology, risk-groups, awareness and brief interventions are explained and discussed.

The online tailored feedback module is newly developed using the TailorBuilder software (OSE, the Netherlands), which uses an assessment questionnaire, a feedback library and algorithms to select the feedback message that matches the answer to each item on the assessment questionnaire and provides tailored feedback. The Feedback module is delivered via a website where participants can log in with personal codes. First, participants fill out a questionnaire and select all barriers which are applicable to them from a list, such as 'I think that alcohol screening and delivery of brief advice is not part of my role as a GP'. Then more specific questions about their motivation, knowledge, ASBI behaviour and about social influences are asked. Personalized feedback is provided on all barriers related to their motivation, knowledge and ASBI behaviour. The tailored feedback module has been pretested among GPs, health promotion researchers and prevention workers and was adjusted when necessary.

An informative website about ASBI implementation is developed in which information about guideline recommendations, the E-learning module, the tailored feedback module as well as information about contacts (e.g. addiction prevention workers) is provided. A referral tool, the
AUDIT-C screening questionnaire as well as a step-wise description of ASBI can be found on the website.

\subsection{Step 4: intervention evaluation}

The aim of the evaluation study is to investigate 1) the effect of the theory-based ASBI implementation program on screening and brief intervention delivery behaviour of GPs (implementation effectiveness), and 2) the effect of the program on patient alcohol consumption (treatment effectiveness).

\subsection{Trial design: a two-arm cluster randomised controlled trial}

The evaluation study is designed as a two-arm cluster randomised controlled trial (Fig. 1). GPs will be recruited and randomised at the practice level into an ASBI implementation program condition or a control condition. In the ASBI implementation program condition GPs will receive the ASBI implementation program as described above. GPs in the control condition will continue care as usual (CAU). GP screening and brief intervention activity will be measured at two time periods: a two-week baseline period (T1) and a two-week post-implementation period (T3). The three-week implementation period (T2) is between the baseline and post-implementation periods. The measurement periods were found to be most feasible for participating GPs and sufficiently long to measure GP alcohol screening and brief intervention delivery behaviour.

Alcohol use of patients with excessive or problematic alcohol use will be assessed at one month and three months after they had the GP consultation. A comparison will be made of the proportion of patients from both conditions (implementation program vs. control) (Fig. 1) who reduced their levels of alcohol consumption to low-risk levels after one-month and three-months.

\subsection{Participants}

The research population consists of [1] GPs working in clinical practice in the province of Limburg in The Netherlands and [2] patients who were asked about their alcohol use during consultation with their GP. Initially, due to practical reasons practices based in the province of Limburg will be approached. All patients who were asked about their alcohol use during consultation will be invited to participate in the study. In order to be eligible to participate in this study, GPs must meet the following criteria: [1] working in general practice; [2] working in co-operation with a practice nurse mental health. The inclusion of the practice nurse mental health is necessary in order to provide GPs with a lowthreshold referral option for patients. There are no exclusion criteria.

\subsection{Randomisation and treatment allocation}

The unit of randomisation will be general practices. Simple randomisation does not guarantee balance in numbers between the two trial-arms. Therefore, we used block randomisation with randomly permuted blocks. Practices will be allocated to a group in blocks to insure that equal number of practices have been assigned to each group. Randomisation will be conducted by using a pseudo-random number generator (www.randomization.com) that generates a random allocation sequence. Allocation concealment is carried out by securing and storing the allocation sequence by an independent research assistant (unaffiliated with the research team). Proper allocation concealment will be ensured, as the research assistant will not release the randomisation code until the practice has been recruited into the trial, which takes place after the baseline measurements of the participating GPs in the practice have been completed.

In block randomisation a study could be compromised if block sizes are known [28], as due to too short block sizes allocation can be guessed. Someone keeping careful track would know the treatment that would 


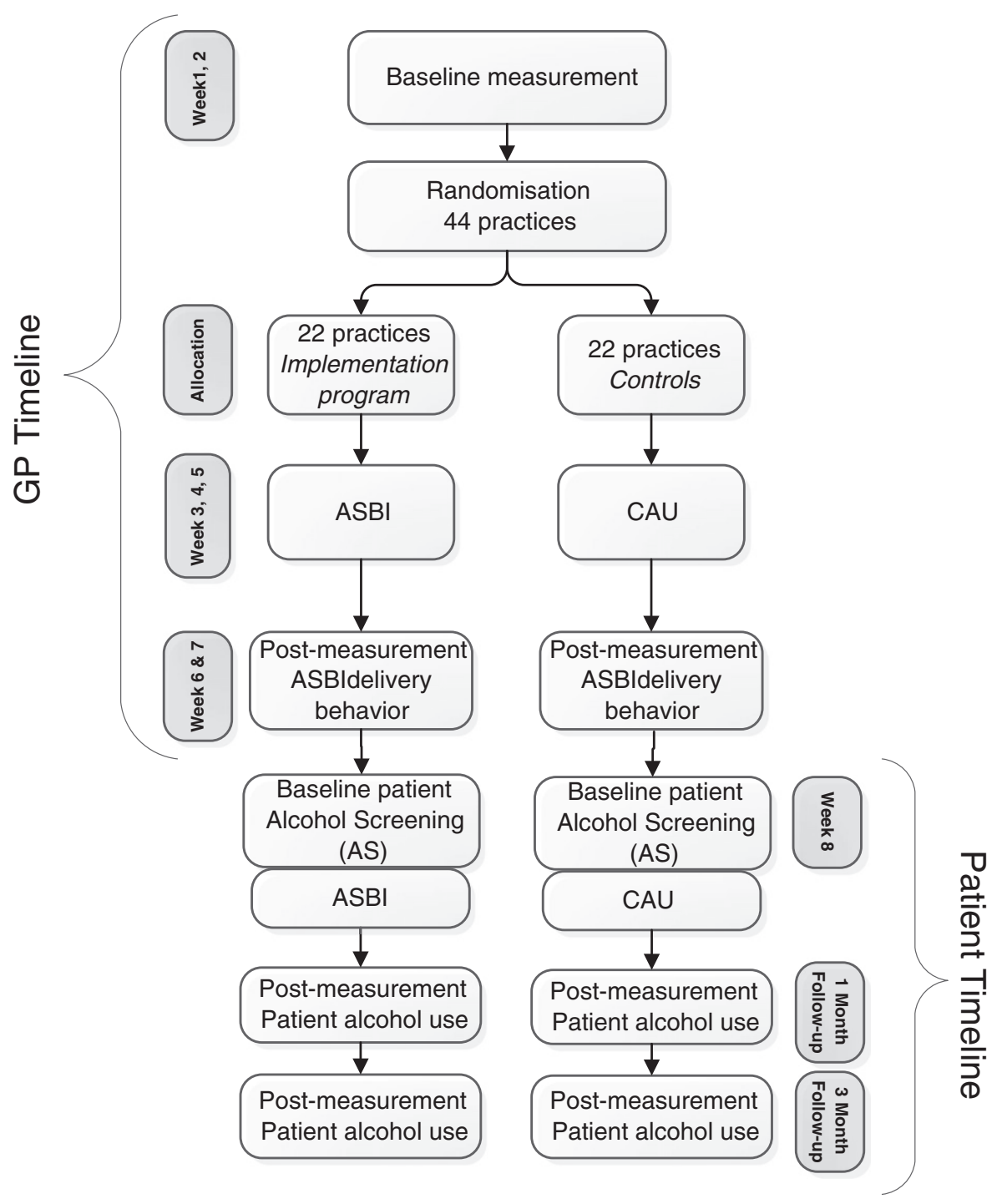

Fig. 1. Study design.

be given to the remaining members of a block once there is only one treatment left to be assigned. Therefore, random block sizes are used to keep the investigators blind to the size of each block. We specified the numbers of practices per block (randomly $4,6,8$ or 10) and the number of each type of block desired [3-2-2-2] resulting in a randomisation sequence for 60 practices randomised into 9 blocks.

\subsection{Study procedure}

\subsubsection{GPS}

Upon enrolment into this study GPs will receive an information letter and an informed consent document to be signed. If the GP decides to take part, written informed consent will be obtained. All GPs and practices will be informed that they can refuse to participate without giving a reason for doing so, and anyone who has already consented can withdraw from the study at any time without providing a reason for doing so.

Participating GPs enter the two-week baseline period, where screening behaviour and intervention delivery behaviour will be registered. GPs in both conditions will be asked to carefully register their ASBI delivery behaviour in the AUDIT-C registration forms, specifically how much the patient drinks and whether the patient received brief advice or was referred to the practice nurse mental health, primary care psychologist, or specialized addiction care (secondary care). Furthermore, baseline measures of demographics, self-reported ASBI delivery and socio-cognitive factors will be measured via online questionnaires.

After the baseline period, practices will be randomised into one of the two study arms. GPs in the implementation condition will enter the implementation phase and will receive the implementation program as described in the previous section. The E-learning module and Feedback module take together approximately 150 minutes that can be completed within 3 weeks (implementation period). Regular email reminders will be sent to promote (re)visit of the intervention modules. GPs in the control condition will continue to deliver care as usual.

\subsubsection{Patients}

After the post-measurement phase of the GP implementation trial the patient-study starts. GPs will hand-out information packages to all patients who are asked about their alcohol use. The GP will ask the patient to read the information about the study and decide about whether to participate in the study at home. Patients can register for the study either by sending an informed consent form or by registering online. Upon registration, patients will receive the questionnaire at home. This procedure has been chosen for two reasons: first to secure patients' time to consider participating in the study at home without feeling rushed into signing forms during the consultation with their GP and 
second, to take account of the fact that GPs in general have little time during consultation to explain about an ongoing study.

\subsection{Sample size calculation}

This trial is designed to detect a $5 \%$ increase of screening rates due to the implementation program, assuming a $6 \%$ screening rate in the control condition $[13,29]$. To detect this difference at a $5 \%$ significance level with $80 \%$ power (using a two sided test), accounting for potential cluster effects by assuming that $95 \%$ of the GP specific screening rates in the control condition vary from $1 \%$ to $12 \%$ (with equal amounts of variation due to practices and due to GPs within a practice), and assuming a cluster size of 2 GPs per practice and 200 patients per GP, 15 GP practices per treatment condition are required. Adjusting for the variation in the number of GPs per practice and the number of patients per GP (leading to a $10 \%$ increase of the number of practices [30]) and expecting a dropout of $20 \%$ of the practices, the aim is to recruit 22 practices per condition. Based on this sample size calculation a total number of 44 participating practices should be large enough to provide a reliable answer to our research question.

This study also aims to detect a $13 \%$ decrease in the percentage of excessive and problem drinkers in the treatment condition compared to CAU (i.e. percentage of patients with negative status on AUDIT-C, i.e. changing from excessive/problematic drinkers to non-excessive/nonproblematic drinkers) [31]. To detect a $13 \%$ decrease at the 5\% significance level with $80 \%$ power (using a two sided test), accounting for cluster effects by assuming that $95 \%$ of the GP specific success rates in the CAU condition vary from $0.5 \%$ to $25 \%$ (with equal amounts of variation due to practices and due to GPs within a practice and an average success rate of 5\%) and assuming a cluster size of 2 GPs per practice and 18 patients per GP, 11 practices per condition are required. Adjusting for the variation in the amount of GPs per practice and patients per GP (leading to a $10 \%$ increase of the number of practices [30]) and expecting a dropout of $20 \%$ of the practices, the aim is to recruit 17 practices per condition. A sample size of 44 practices, as required for detecting a difference of $5 \%$ in screening rates, is therefore also sufficient to detect a $13 \%$ decrease in the percentage of problematic drinkers.

\subsection{Outcome measures and measurement instruments}

\subsubsection{Screening rate}

The screening rate will be expressed as the absolute increase in screening using the Alcohol Use Disorder Identification Test (AUDIT-C [32]), The AUDIT-C will be used as a screening tool and consists of the following three questions: [1] how often alcohol is consumed (never, once a month, 2 to 4 times a month, 2 to 3 times a week, 4 times a week or more); [2] how many glasses of alcohol are typically consumed during a drinking day ( 1 to 2 glasses, 3 to 4 glasses, 5 to 6 glasses, 7 to 9 glasses or at least 10 glasses) and [3] how often does the patient drink at least four (for women) and at least five (for men) glasses per occasion (never, at most once a month, monthly, weekly or daily or almost daily). The total score ( 0 to 12 points) from the AUDIT-C questionnaire will be calculated by summing the values ( $0-4$ points) for each of the three questions. The AUDIT-C is equal to the AUDIT in terms of validity and reliability in primary health care [33]. We will use the recommended cut-off points for problematic alcohol use at $\geq 5$ points for men and $\geq 4$ points for women. In order to investigate the symptom-specific screening rate, GPs also specify on the AUDIT-C form which symptom(s) the patient had.

\subsubsection{Detection rate}

The detection rate will be expressed as the proportion of patients who screen positive on the AUDIT-C divided by the total number of patients screened.

\subsubsection{Rate of brief intervention delivery}

The brief intervention rate is expressed as the proportion of patients who receive a brief advice or referral to another professional divided by the total amount of patients who screen positive on the AUDIT-C. Brief intervention delivery will be measured with an extra question on the AUDIT-C questionnaire, which inquires about the course of action taken by the GP ("What treatment have you given or what course of action did you undertake? Brief advice, referral to practice nurse, referral to psychologist, referral to specialized addiction care, otherwise...").

\subsubsection{Self-reported ASBI delivery}

In an online questionnaire GPs are given two statements regarding their ASBI delivery behaviour, which they have to rate on a 7-point Likert scale with response categories ranging from ' 1 ' (strongly agree) to '7' (strongly disagree). The two following statements are provided: 'I ask about alcohol use of every patient with symptoms which might be related to excessive or problematic alcohol (e.g.: frequent visits and a changing, unclear pattern of symptoms or unexplained somatic symptoms; gastro-intestinal problems; psychological symptoms)' and 'I give each patient who consumes too much alcohol advice about alcohol use'.

\subsubsection{Patient alcohol consumption}

Change in patient alcohol consumption is expressed as the proportion: patients who score $\geq 5$ for men or $\geq 4$ for women on the AUDIT-C who reduced their levels of alcohol consumption to low-risk level divided by the total amount of patients who score $\geq 5$ for men or $\geq 4$ for women on the AUDIT-C.

\subsection{Covariates and intermediate outcome measures}

Assessment of co-variates and intermediate outcome measures include:

GP demographics: gender, age, working hours, hours of training in alcohol problems before start of study and own alcohol use, practice type and level of urbanization. These data will be collected from a self-report questionnaire at baseline.

Patient demographics: age, gender, ethnicity, household composition, educational level, smoking status, co-morbidity. This will be assessed using a self-report questionnaire at baseline.

The Short Alcohol and Alcohol Problems Perception Questionnaire (SAAPPQ) [34]: The SAAPQ is a validated questionnaire which will be used as a measure for GPs' beliefs about capabilities (i.e. role adequacy and task-specific self-esteem), beliefs about professional role (i.e. role legitimacy), motivation and work satisfaction.

Stigmatizing beliefs: GPs' social stigma beliefs are measured on a 7point Likert scale with response categories ranging from ' 1 ' (strongly agree) to '7' (strongly disagree) (e.g.: 'I think that excessive and problematic alcohol users are difficult patients who don't want to change their behaviour', etc.).

Knowledge: The questions used to assess GPs' knowledge are measured on a 7-point Likert scale with response categories ranging from ' 1 ' (strongly agree) to '7' ( strongly disagree) (e.g. 'I know what excessive and problematic alcohol use is'; 'I know which symptoms can be early signs of excessive or problematic alcohol use', etc.).

\section{Analysis}

Due to the hierarchical structure of the data (three-level hierarchical structure: providers nested within practices and patients nested within providers), we will perform multilevel regression analyses with practices and providers as random factors. For the primary outcome measures (ASBI or not, excessive/problem drinker or not), mixed logistic regression will be done to test for the effect of ASBI versus CAU. Multilevel regression allows for modelling heterogeneity at all levels and takes into account the inter-dependency of scores within practices and providers at all times of follow-up. 
Since different practice types are included in this study, we will adjust for practice type by including this variable as a covariate in our analyses. To investigate the usability and perceived quality of all parts of the implementation program descriptive analyses as well as linear and logistic multilevel regression analyses will be conducted. To investigate whether effects of the implementation intervention on ASBI behaviour are mediated by cognitive variables (i.e. attitude; SAAPPQ scores, stigmatizing beliefs and knowledge level) mediation-analyses will be done as secondary analyses.

\section{Process evaluation}

During post-measurement, all GPs are given an online process evaluation questionnaire in order to assess the utilisation and perceived quality of all parts of the implementation program. The program will be evaluated on user-friendliness, overall appreciation, practicability and feasibility. All participants are given the opportunity to provide an online qualitative assessment of the program and suggestions for improvement.

In the patient study, patients' attitudes toward discussing alcohol use with their GP the quality of the GP's screening and alcohol advice will be evaluated. A questionnaire based on a previously used assessment by Nilsen and colleagues [35] will be used. Questions included are among others: 'I did not mind that my GP asked me about my alcohol use'; 'My GP asked me about my alcohol use in a neutral way', 'My GP made the link to my symptoms and explained how my symptoms might be related to alcohol use', 'I think my GP provided me with clear advice about alcohol use', 'I think my GP provided me with clear advice about the care/referral that I need regarding my alcohol use'. The statements have to be rated on a 7-point Likert scale with response categories ranging from ' 1 ' (strongly agree) to ' 7 ' (strongly disagree). These questions will be added to the first post-test questionnaire for the patients.

\section{Discussion}

This paper described the step-wise development and evaluation protocol of a theory-based implementation program aimed at increasing ASBI delivery in general practices in The Netherlands. In addition, the implementation program is aimed at reducing patient alcohol consumption. The program is based on the identification of underlying factors and subsequent mapping and application of evidence-based behaviour change techniques. The implementation program covers previously identified barriers related to motivation, capability and opportunity and provides a complete package which GPs can follow online at their convenience. Our program is distinctive from previous initiatives in that it uses new methods for implementation (e-learning and tailored feedback), which haven't been investigated yet in the field of ASBI implementation. Moreover, the program is strongly theory-based as well as practice-based in the sense that the implementation program components have been developed in line with preferences and feasibility ratings of GPs reported in a previous Delphi study [18]. The use of theory to inform the development of behaviour change and implementation interventions is strongly advocated by experts in the field of implementation science [15]. Theory helps in identifying the most critical factors that contribute to the implementation of an innovation and provides insight into the most appropriate methods that can be used to change these critical factors and facilitate implementation [15]. In addition, a practice-based approach should ensure that ASBI implementation is adapted to local circumstances to make implementation feasible [16]. We hypothesize, that GPs who receive the implementation program will increase their screening and brief advice delivery behaviour consequently leading to a higher proportion of patients who decrease their alcohol consumption.
GPs participating in ASBI trials evaluating its effects are more often than not highly trained and supervised by research staff delivering ASBI under optimal conditions, which is typical for efficacy trials [36]. Consequently, previous trials which found an effect of ASBI have been criticised as not being representative of real-world clinical conditions and the current literature has been criticised as not being very informative about whether ASBI works under real-world conditions [9]. Therefore, the actual effectiveness of ASBI in real-world settings as opposed to the efficacy of ASBI evaluated in tightly controlled efficacy trials has recently been subject to extensive debate [39]. Moreover null findings of previous implementation trials (e.g. SIPS trial; van Beurden trial) have been attributed to lack of fidelity in the implementation of ASBI in large, complex, cluster randomised trials.

Therefore, rather than being carried out under selected, ideal circumstances, this cluster randomised controlled trial has a pragmatic approach to investigate how ASBI under real-world conditions can be implemented and whether our program has an effect on patient alcohol consumption. A pragmatic approach recognizes that in real world conditions the healthcare system is a multilevel, complex, and interacting system that changes over time and requires flexibility and adaptation [37]. To ensure the pragmatic nature of the trial, we included the following criteria for a pragmatic trial as suggested by Thorpe et al. [38]: [1] to ensure feasibility, GPs have been actively involved in the program development and design of the study, [2] the selection of applicable implementation strategies of the program is based on the preparatory Delphi study conducted among GPs, practice nurses and addiction prevention experts [18], [3] the target behaviour (ASBI delivery) is adapted to a feasible standard (i.e. symptom-specific screening and delivery of brief advice rather than universal screening and delivery of prolonged interventions) in line with the NHG Guideline Problematic Alcohol Use as specified by the Dutch College of General Practitioners, [4] no formal strategy will be used to monitor or encourage adherence to the study protocol, [5] the restrictiveness of the trial inclusion criteria are limited [6] researchers as well as addiction prevention experts will be encouraged to adopt a passive approach and lessen influence in the general practice setting as well as in the implementation process (i.e. GPs contact the research team for queries rather than actively contacting the GPs), [7] GPs in the control group are allowed to provide patients with any type of care from any healthcare professional (i.e. care as usual).

Our trial also has limitations. This trial does not include objective measurements of GP behaviour (e.g. use of registration data), but relies on self-reported documentation on AUDIT-C forms when inquiring patients about alcohol. This method was chosen because GPs do not always register inquiries about alcohol or even diagnoses of problematic alcohol use in the medical records. Therefore, registration data have been shown to be unreliable and to not provide an accurate picture of what is inquired during consultations with patients. Based on previous implementation trials [13] a high drop-out rate can be expected. However, previous trials used extensive implementation programs requiring a lot of time and dedication from GPs. In contrast to this, the current implementation program is of short duration and has been made available online. The program has also been made personally relevant by tailoring feedback to the needs and barriers of each GP. Moreover, to encourage response we will send reminders after two and four weeks.

This paper adds to the literature by providing in-depth information of the stepwise development and evaluation of the implementation program. The current ASBI implementation program relies on a solid theoretical foundation which makes the core components of the program explicit. This trial provides new knowledge that can be used in national and international ASBI implementation initiatives designed for widespread use in general practice settings. Moreover, when shown effective, the program can easily be adapted to other health professionals involved in 
ASBI and can be made widely available and imbedded in healthcare systems.

\section{List of abbreviations used}

$\begin{array}{ll}\text { GP } & \text { General Practitioner } \\ \text { ASBI } & \text { Alcohol Screening and Brief Intervention } \\ \text { BCW } & \text { Behaviour Change Wheel } \\ \text { TDF } & \text { Theoretical Domains Framework } \\ \text { COM-B } & \text { Capability, Opportunity, Motivation - Behaviour } \\ \text { AUDIT-C } & \text { The Alcohol Use Disorder Identification Test } \\ \text { SAAPPQ } & \text { The Short Alcohol and Alcohol Problems Perception } \\ & \text { Questionnaire }\end{array}$

\section{Ethical approval}

This trial (METC 15-4-161) is reviewed by the Medical Ethical Committee of the Academic Hospital Maastricht/Maastricht University who declared that no medical ethical clearance for this study was needed according to the rules of the Medical Research Involving Human Subjects Act (WMO). This study will be conducted in accordance with the Code of Conduct for the Use of Data in Health Research of the Federation Medical Scientific Union (FMWV) and the Dutch Act on the Protection of Personal Data.

\section{Competing interests}

The authors declare that they have no conflict of interest.

\section{Authors' contributions}

L.A., A.O., and D.v.d.M. developed the implementation program and the study design of this trial. M.C. additionally helped the authors with planning the statistical analyses and the sample size calculations. L.A. wrote the first draft of the manuscript, and all co-authors provided critical feedback. All authors read and approved the final manuscript.

\section{Acknowledgements}

This study was financed by Mondriaan Institute for Mental Health and CAPHRI School of Public Health and Primary Care. The authors would like to thank Wilma Noteborn (Lionarons GGZ), Marjan van den Akker, Jos Boesten and all members of Registratie Netwerk Huisartsen (department of Family Medicine, Maastricht University) for their valuable input in the design of this study.

\section{References}

[1] WHO, Global Status Report on Alcohol and Health 2014, World Health Organization, Geneva, 2014.

[2] S.S. Lim, T. Vos, A.D. Flaxman, G. Danaei, K. Shibuya, H. Adair-Rohani, et al., A comparative risk assessment of burden of disease and injury attributable to 67 risk factors and risk factor clusters in 21 regions, 1990-2010: a systematic analysis for the Global Burden of Disease Study 2010, Lancet 380 (9859) (2012) 2224-2260.

[3] D. Van Dijck, R.A. Knibbe, De Prevalentie van Probleemdrinken in Nederland, Universiteit Maastricht, Maastricht, 2005.

[4] L.J. Boomsma, I.M. Drost, I.M. Larsen, J.J.H.M. Luijkx, G.J. Meerkerk, N. Valken, et al., NHG-Standaard Problematisch alcoholgebruik (Derde herziening), Huisarts Wet. 57 (12) (2014) 638-646.

[5] E. Burgering, H. Willems, Arts vraagt te weinig naar drankgebruik, Dokter \& Patient. 68 (2013) 795-797.

[6] E.F. Kaner, H.O. Dickinson, F. Beyer, E. Pienaar, C. Schlesinger, F. Campbell, et al., The effectiveness of brief alcohol interventions in primary care settings: a systematic review, Drug Alcohol Rev. 28 (3) (2009) 301-323.

[7] A. O'Donnell, P. Anderson, D. Newbury-Birch, B. Schulte, C. Schmidt, J. Reimer, et al. The impact of brief alcohol interventions in primary healthcare: a systematic review of reviews, Alcohol Alcohol. 49 (1) (2014) 66-78.
[8] M.F. Fleming, M.P. Mundt, M.T. French, L.B. Manwell, E.A. Stauffacher, K.L. Barry, Brief physician advice for problem drinkers: Long-term efficacy and benefit-cost analysis, Alcohol. Clin. Exp. Res. 26 (1) (2002) 36-43.

[9] R. Saitz, The best evidence for alcohol screening and brief intervention in primary care supports efficacy, at best, not effectiveness: you say tomato, I say tomato? That's not all it's about, Addict. Sci. Clin. Pract. 9 (2014) 14

[10] M. Johnson, R. Jackson, L. Guillaume, P. Meier, E. Goyder, Barriers and facilitators to implementing screening and brief intervention for alcohol misuse: a systematic review of qualitative evidence, J. Public Health 33 (3) (2011) 412-421.

[11] E.C. Williams, M.L. Johnson, G.T. Lapham, R.M. Caldeiro, L. Chew, G.S. Fletcher, et al., Strategies to implement alcohol screening and brief intervention in primary care settings: A structured literature review, Psychol. Addict. Behav. 25 (2) (2011) 206-214.

[12] M. Keurhorst, I van de Glind, B. do Amaral-Sabadini M, P. Anderson, E. Kaner, D. Newbury-Birch, et al., Implementation strategies to enhance management of heavy alcohol consumption in primary health care: a meta-analysis, Addiction 110 (12) (2015) 1877-1900.

[13] I. Van Beurden, P. Anderson, R.P. Akkermans, R.P.T.M. Grol, M. Wensing, M.G.H. Laurant, Involvement of general practitioners in managing alcohol problems: A randomized controlled trial of a tailored improvement programme, Addiction 107 (9) (2012) 1601-1611.

[14] S.D. French, S.E. Green, D.A. O'Connor, J.E. McKenzie, J.J. Francis, S. Michie, et al., Developing theory-informed behaviour change interventions to implement evidence into practice: a systematic approach using the theoretical domains framework, Implement. Sci. 7 (2012) 38

[15] R. Grol, M. Wensing, M. Eccles, D. Davis, Improving Patient Care: the Implementation of Change in Health Care, Second Edition John Wiley \& Sons L, 2013 (editor).

[16] R. McCormick, B. Docherty, L. Segura, J. Colom, A. Gual, P. Cassidy, et al., The research translation problem: Alcohol screening and brief intervention in primary care - real world evidence supports theory, Drug-Educ. Prev. Polic. 17 (6) (2010) 732-748.

[17] S. Michie, M.M. van Stralen, R. West, The behaviour change wheel: a new method for characterising and designing behaviour change interventions, Implement. Sci. 6 (2011).

[18] L. Abidi, A. Oenema, P. Nilsen, P. Anderson, D. van de Mheen, Strategies to overcome barriers to implementation of alcohol screening and brief intervention in general practice: a Delphi study among healthcare professionals and addiction prevention experts, Prev. Sci. (2016)

[19] S. Michie, M. Johnston, J. Francis, W. Hardeman, M. Eccles, From theory to intervention: mapping. Theoretically derived behavioural determinants to behaviour change techniques, Appl. Psychol. 57 (4) (2008) 660-680.

[20] M. Porcheret, C. Main, P. Croft, R. McKinley, A. Hassell, K. Dziedzic, Development of a behaviour change intervention: a case study on the practical application of theory, Implement. Sci. 9 (1) (2014) 42.

[21] J.G. Ruiz, M.J. Mintzer, R.M. Leipzig, The impact of E-learning in medical education, Acad. Med. 81 (3) (2006) 207-212.

[22] R. Kulier, S.F. Coppus, J. Zamora, J. Hadley, S. Malick, K. Das, et al., The effectiveness of a clinically integrated e-learning course in evidence-based medicine: a cluster randomised controlled trial, BMC Med. Educ. 9 (2009) 21.

[23] D.A. Cook, A.J. Levinson, S. Garside, Time and learning efficiency in Internet-based learning: a systematic review and meta-analysis, Adv. Health Sci. Educ. Theory Pract. 15 (5) (2010) 755-770.

[24] M.K. Campbell, I. Tessaro, B. DeVellis, S. Benedict, K. Kelsey, L. Belton, et al., Effects of a tailored health promotion program for female blue-collar workers: health works for women, Prev. Med. 34 (3) (2002) 313-323.

[25] H. de Vries, S.P. Kremers, T. Smeets, J. Brug, K. Eijmael, The effectiveness of tailored feedback and action plans in an intervention addressing multiple health behaviors, Am. J. Health Promot. 22 (6) (2008) 417-425.

[26] M.L. Lustria, S.M. Noar, J. Cortese, S.K. Van Stee, R.L. Glueckauf, J. Lee, A meta-analysis of web-delivered tailored health behavior change interventions, J. Health Commun. 18 (9) (2013) 1039-1069.

[27] Contact M.

[28] J. Efird, Blocked randomization with randomly selected block sizes, Int. J. Environ. Res. Public Health 8 (1) (2011) 15-20.

[29] M. Funk, S. Wutzke, E. Kaner, P. Anderson, L. Pas, R. McCormick, et al., A multicountry controlled trial of strategies to promote dissemination and implementation of brief alcohol intervention in primary health care: findings of a World Health Organization collaborative study, J. Stud. Alcohol 66 (3) (2005) 379-388.

[30] M.J. Candel, G.J. Van Breukelen, Sample size adjustments for varying cluster sizes in cluster randomized trials with binary outcomes analyzed with second-order PQL mixed logistic regression, Stat. Med. 29 (14) (2010) 1488-1501.

[31] A. Moyer, J.W. Finney, C.E. Swearingen, P. Vergun, Brief interventions for alcoho problems: a meta-analytic review of controlled investigations in treatmentseeking and non-treatment-seeking populations, Addiction 97 (3) (2002) 279-292.

[32] K. Bush, D.R. Kivlahan, M.B. McDonell, S.D. Fihn, K.A. Bradley, The AUDIT alcohol consumption questions (AUDIT-C): an effective brief screening test for problem drinking. Ambulatory Care Quality Improvement Project (ACQUIP). Alcohol Use Disorders Identification Test, Arch. Intern. Med. 158 (16) (1998) 1789-1795

[33] A. Gual, L. Segura, M. Contel, N. Heather, J. Colom, Audit-3 and audit-4: effectiveness of two short forms of the alcohol use disorders identification test, Alcohol Alcohol. 37 (6) (2002) 591-596. 
[34] P. Anderson, S. Clement, The AAPPQ revisited: the measurement of general practitioners' attitudes to alcohol problems, Br. J. Addict. 82 (7) (1987) 753-759.

[35] P. Nilsen, P. Bendtsen, J. McCambridge, N. Karlsson, K. Dalal, When is it appropriate to address patients' alcohol consumption in health care-national survey of views of the general population in Sweden, Addict. Behav. 37 (11) (2012) 1211-1216.

[36] B.R. Flay, A. Biglan, R.F. Boruch, F.G. Castro, D. Gottfredson, S. Kellam, et al., Standards of evidence: criteria for efficacy, effectiveness and dissemination, Prev. Sci. 6 (3) (2005) 151-175.
[37] R. Kessler, R.E. Glasgow, A proposal to speed translation of healthcare research into practice dramatic change is needed, Am. J. Prev. Med. 40 (6) (2011) 637-644.

[38] K.E. Thorpe, M. Zwarenstein, A.D. Oxman, S. Treweek, C.D. Furberg, D.G. Altman, et al., A pragmatic-explanatory continuum indicator summary (PRECIS): a tool to help trial designers, CMAJ 180 (10) (2009) E47-E57.

[39] N. Heather, The efficacy-effectiveness distinction in trials of alcohol brief intervention, Addict. Sci. Clin. Pract. 9 (2014) 13. 